\title{
Kupffer cells in immune activation and tolerance toward HBV/HCV infection
}

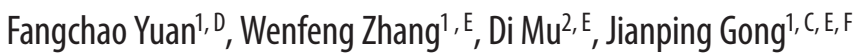 \\ ${ }^{1}$ Chongqing Key Laboratory of Hepatobiliary Surgery and Department of Hepatobiliary Surgery, Second Affiliated Hospital of Chongqing Medical University, Chongquing, China \\ ${ }^{2}$ Department of Infectious Diseases, Institute for Viral Hepatitis, Key Laboratory of Molecular Biology for Infectious Diseases, Ministry of Education, Second Affiliated Hospital of \\ Chongqing Medical University, Chongquing, China
}

A - research concept and design; B - collection and/or assembly of data; $\mathrm{C}$ - data analysis and interpretation;

$D$ - writing the article; $E$ - critical revision of the article; $F$ - final approval of article

\author{
Address for correspondence \\ Jianping Gong \\ E-mail:18581321179@163.com

\section{Funding sources} \\ The review was supported by the National \\ Natural Science Foundation of China \\ (grant no. 81370580). \\ Conflict of interest \\ None declared

\section{Acknowledgements} \\ The figure accompanying this review was \\ created and kindly provided by Xiao Ke from \\ Peking Union Medical College (Beijing, China).
}

\begin{abstract}
Kupffer cells (KCS) are macrophages that are found in the sinusoids of the liver. KCs are a crucial part of the innate immune system, acting as scavengers and phagocytes. KCs and sinusoidal endothelial cells together form the first immune barrier of the portal system. Studies show that KCs can not only maintain homeostasis in the immune response, but also facilitate the pathogenesis of type B and type C hepatitis (HBV/HCV) through their antigen-presenting function and secretion of soluble mediators. KCs can express toll-like receptors (TLRs), Fas ligand (FasL) and programmed cell death ligand 1 (PD-L1), and secrete large amounts of inflammatory factors leading to immune tolerance toward HBV/HCV. On the one hand, KCs contribute to the clearance of HBV/HCV due to their nature as innate immune cells. At the same time, KCs induce immune tolerance toward $\mathrm{HBV} / \mathrm{HCV}$, which leads to chronicity of the infection. The dual role of KCs in the immune response toward HBV/HCV means it is a gigantic challenge for scientists to illuminate the detailed mechanisms involved, but it also offers important potential therapeutic targets.
\end{abstract}

Key words: HBV, HCV, Kupffer cell

Received on July 14, 2015

Revised on August 12, 2015

Accepted on April 20, 2016

DOI

$10.17219 /$ acem/62759

\section{Copyright}

Copyright by Author(s)

This is an article distributed under the terms of the

Creative Commons Attribution Non-Commercial License

(http://creativecommons.org/licenses/by-nc-nd/4.0/) 
Worldwide, more than 500 million people are suffering from chronic infection with type $B$ or type $C$ hepatitis (HBV/HCV), especially in developing countries. ${ }^{1}$ Such infections may gradually induce liver fibrosis and cirrhosis, and eventually hepatocellular carcinoma among the infected patients. Research into the pathogenesis of HBV/ $\mathrm{HCV}$ is urgently needed.

Kupffer cells (KCs) are among the innate immune cells found in the liver. KCs make up 80-90\% of macrophages and compose $15 \%$ of the cells in the liver. KCs are a crucial part of the systemic mononuclear phagocyte system, playing a significant role in the development of liver diseases. As an innate immune cell, KCs are capable of pathogen capturing, recruiting additional immune cells and antigen-presenting cells that are essential in the clearance of $\mathrm{HBV} / \mathrm{HCV}^{2}$ This is the beneficial side of KCs in the immune response to $\mathrm{HBV} / \mathrm{HCV}$. However, studies have also proven the role of $\mathrm{KCs}$ in inducing immune tolerance toward $\mathrm{HBV} / \mathrm{HCV}^{3}$ The dual role of $\mathrm{KCs}$ in the immune response to $\mathrm{HBV} / \mathrm{HCV}$ infection makes it difficult for scientists to thoroughly study the mechanisms involved, and KCs are inevitably part of the study of the pathogenesis of $\mathrm{HBV} / \mathrm{HCV}$.

\section{The immune response mediated by $\mathrm{KC}$ s in $\mathrm{HBV} / \mathrm{HCV}$ infection}

Little evidence has been reported to elucidate the mechanism by which $\mathrm{HBV} / \mathrm{HCV}$ particles may stimulate KCs. The complicated protein structure of $\mathrm{HBsAg}$ is conducive to $\mathrm{KCs}$ and epithelial cells up-taking the hepatitis B virus, after which KCs initiate clearance of virus. Scientists have tested liver samples from patients chronically infected with $\mathrm{HBV} / \mathrm{HCV}$, and the number of KCs observed is apparently larger than in healthy people. ${ }^{4}$ Furthermore, exposing KCs to HBsAg in vitro can cause stronger production of pro-inflammatory cytokines. ${ }^{5}$ This is especially true in the hepatic portal area. In the immune-active phase of chronic hepatitis B infection KCs are much more numerous than in the immune-suppression phase. When compared with splenic and peritoneal macrophages, KCs outweigh them in the ability to up-take particles in circulation and can produce more reactive oxygen species (ROS). ${ }^{6}$ The existing evidence shows that KCs may be able to control HBV/HCV infection through cytokine production, synergistic effects with other cells and mediation of virus-specific immunity.

\section{$\mathrm{KC}$ receptors activated during HBV/HCV Infection}

Macrophages have been shown to express scavenger receptors, toll-like receptors (TLRs), RIG-like receptors (RLRs), NOD-like receptors (NLRs) and C-type lectins both in vivo and in vitro. ${ }^{7}$ However, few studies have been carried out to clarify whether such receptors are also expressed by KCs. In human, rat and mice KCs, the expression of C-type lectins and scavenger receptors closely related to the mediation of phagocytosis has been confirmed. ${ }^{8}$ Human KCs have been proven to express TLR 2-4, while expression of TLR1-9 and retinoic-acid-inducible geneI(RIG-I) have been detected in KCs from rats and mice. ${ }^{9,10} \mathrm{KCs}$ also express mannose receptors and CD14. ${ }^{3}$ The detailed mechanisms of the interaction between $\mathrm{KC}$ receptors and $\mathrm{HBV} / \mathrm{HCV}$ viral protein still remain to be elucidated. Although researchers have not found any direct evidence from KCs, studies on THP-1 monocytes, monocytes and dendritic cells have shown that surface receptors binding with HBV or HBV proteins will eventually activate the cells through TLRs, HSPG, CD14 and mannose receptors. ${ }^{11}$ For example, HBcAg can be recognized by THP-1 monocytes through TLR2 and heparan sulfate proteoglycan (HSPG), and the activated THP-1 cell will produce interleukin-6 (IL-6), IL-12p40 and tumor necrosis factor-alpha (TNF- $\alpha){ }^{11,12}$

The mechanism of the interaction between $\mathrm{HCV}$ and KCs has been elucidated more clearly than the mechanism of HBV infection. HCV infects hepatocytes through binding with HSPG, low-density lipoprotein receptors and scavenger receptor $\mathrm{B} 1$, which are also expressed by KCs. HCV-E2 binding to KCs is CD81-dependent when it is co-incubated with KCs. DC-SIGN is also activated to facilitate the binding of $\mathrm{HCV}$ and $\mathrm{KCs}$. HCV can activate KCs to produce cytokines such as IL-1及, IL-6, IL-10 and TNF- $\alpha$, through TLR2 and TLR4. ${ }^{13}$

\section{Signaling pathway activation during HBV infection}

Further studies on the signaling pathways activated during HBV infection are still needed. Hösel et al. showed that HBV particles and HBsAg induce CD68+ non-parenchymal cells from the liver to secrete IL-1 $\beta$, IL-6, chemokine (C-X-C motif) ligand 8 (CXCL8) and TNF- $\alpha$ by activating the nuclear factor kappa $\mathrm{B}(\mathrm{NF}-\mathrm{KB})$ pathway. ${ }^{14}$ The IL- 6 and TNF- $\alpha$ secreted is able to inhibit the replication of HBV in hepatocytes, while TNF- $\alpha$ causes no damage to the target cells. ${ }^{15}$ Once KCs recognize HBV, the NF-кB pathway is activated, accompanied by the production of certain inflammatory cytokines, such as IL-6, IL-8, IL-1 and TNF- $\alpha$. The IL- 6 secreted by KCs control HBV gene expression and replication in hepatocytes on the transcription level. Hösel et al. pointed out that the recognition of $\mathrm{HBV}$ by cells like KCs and the accompanying temporary activation of the NF- $\mathrm{kB}$ pathway may ensure homeostasis and the survival of the infected cells, guaranteeing a favorable environment for HBV replication. This is a brand new mechanism to support the replication of $\mathrm{HBV}$ through the production of cytokines. Attenuated early immune response generated by IL- 6 is favorable for $\mathrm{HBV}^{14}$ 


\section{Signaling pathway activation during $\mathrm{HCV}$ infection}

Once CD14+ KCs or other macrophage-derived monocytes are bound to HCV core protein and NS3, they can produce inflammatory cytokines, including IL-1 $\beta$, IL-6, interferon beta (IFN $\beta$ ) and TNF- $\alpha$, to inhibit HCV replication, accompanied by secretion of immune-suppressing mediator IL-10. HCV core protein and a type of ligand of TLR 2 are involved in immune regulation. Tu et al. found that when recombinant $\mathrm{HCV}$ core protein was added to a culture, human KCs would significantly increase the production of IL-1 $\beta$, TNF- $\alpha$ and IL- $10 .{ }^{11}$ After bonding to $\mathrm{HCV}$ core protein, KCs activate a myeloid differentiation molecular 88 (MYD88) dependent pathway to produce a large number of pro-inflammatory cytokines. Meanwhile, HCV core protein can bind to the C1q complement receptor expressed by macrophages and dendritic cells, which leads to a reduction in IL-12 secretion. ${ }^{16}$

The TLR-signaling pathway is also crucial in $\mathrm{KCs}$ ' interaction with HCV. KCs can recognize NS3 through TLR4, while TLR4 can transfer the signaling to KCs, activating NF-KB and promoting secretion of TNF-a. ${ }^{17} \mathrm{KC}$-derived TNF- $\alpha$ could increase the permeability that allows $\mathrm{HCV}$ to enter host cells more easily. Lipopolysaccharide (LPS) could stimulate KCs to produce TNF- $\alpha$, indirectly promoting HCV infection. ${ }^{18}$

\section{Interactions between $\mathrm{KCs}$ and other cells in the immune response}

KCs could indirectly take part in the liver immune response via secretion of related cytokines to recruit leukocytes to the liver and activate them as well, thus interacting with hepatocytes, resident immune cells and infiltrating leukocytes. ${ }^{19}$ CXCL8 produced by KCs can recruit natural killer (NK) cells and NK T cells to the liver in the early stage of infection, and then such cells can be activated by cytokines. ${ }^{20}$ Dendritic cells can also be attracted to the liver by $\mathrm{KCs}$, leading to an antigen-specific $\mathrm{T}$ cell response. KCs present $\mathrm{HBV} / \mathrm{HCV}$ to $\mathrm{CD} 4+$ and CD 8+ T cells through their antigen-presenting function, and promote the production of IFN- $\gamma$. In addition, KCs can express cytotoxic molecules such as TNF-related apoptosis-inducing ligand (TRAIL), Fas ligand (FasL), granzyme B, perforin and ROS, promoting lysis of infected hepatocytes. However, once non-specific KCmediated cytolysis of hepatocytes is initiated, the lysis effect will no longer be limited to infected hepatocytes. ${ }^{21} \mathrm{KC} \mathrm{cy-}$ totoxicity therefore leads to more severe damage to organs.

\section{Immune tolerance mediated by $\mathrm{KCs}$ in $\mathrm{HBV} / \mathrm{HCV}$}

Despite the fact that KCs have the ability to clear the $\mathrm{HBV} / \mathrm{HCV}$ virus, in actual conditions the virus interferes with KCs' pro-inflammatory role to escape the host's immune response. Studies have shown that $\mathrm{HBV} / \mathrm{HCV}$ may interfere with the TLR pathway, the RIG-I pathway and the follow-up pro-inflammatory activity of hepatocytes or immune cells. ${ }^{22}$ Blood-borne pathogens, including $\mathrm{HBV} / \mathrm{HCV}$, take advantages of $\mathrm{KC}$ endocytosis to enter the liver and to escape the host cell's immune system. KCs are continuously exposed to a variety of pathogens from the gut. In order to prevent excessive inflammation that would cause liver damage, $\mathrm{KCs}$ initiate immune tolerance to limit the effect of sustained activation of the immune system. ${ }^{23}$ Pathogen-associated molecular patterns (PAMPs) can activate KCs and promote the secretion of IL- 6 by binding to pattern recognition receptors (PRRs) on the surface of KCs. This process in turn enhances the pathogen-binding ability and phagocytosis of KCs, and reduces the expression of harmful proteins in the acute phase, including the complement system, $\mathrm{C}$ reactive protein and TNF- $\alpha$. The activation of PRRs initiates KCs' expression of IL-10, TGF $\beta$ and prostaglandin. Arginase and indoleamine 2,3-dioxygenase (IDO) from the liver can suppress the reproduction of pathogens, but meanwhile they inhibit the metabolism of amino acids that are crucial to the replication of immune cells. ${ }^{24}$ Overall the expression of PRRs activates innate immunity and allows systemic antibacterial activity, and also limit the local adaptive immune response.

\section{The TLR pathway}

The TLR family plays an indispensable role in the innate immune system of mammals. ${ }^{25}$ At least 10 members of the TLRs family have been discovered, and it has been confirmed that many of them are expressed by KCs. Immune tolerance of HBV mediated by TLRs is mainly realized by 2 mechanisms. One is to reduce TLRs on surface of immune cells, including KCs. The other is to reduce the effect of TLRs in the innate immune system. Visvanathan et al. found reduced expression of TLR2, TLR3 and TLR4 on KCs in HBeAg+ patients. ${ }^{26}$ TLR2-mediated phosphorylation of p38 mitogen-activated protein kinases (MAPKs) and c-Jun N-terminal kinases (JNKs) and the production of IL- 6 , TNF- $\alpha$ and IL-12 were inhibited in monocytes when they were co-incubated with HBsAg and HBeAg. Subsequent experiments demonstrated that the activation level of JNK in monocytes and CD14+ macrophages declined significantly. ${ }^{27}$ It is safe to speculate that through TLRs, HBeAg may down-regulate the surveillance capability in HBV-infected KCs and eventually lead to immune escape. Wu et al. confirmed that TLR3 and TLR4-activated KCs can inhibit the replication of the HBV virus. The antiviral effect of TLR3 is achieved through binding to its ligand and production of IFN $\beta .{ }^{28}$ Although the antiviral effects of KCs can be achieved through the activation of TLRs, $5-10 \%$ of HBV patients still develop the immune tolerant phase. Studies have confirmed that certain viruses could sabotage the host's immune defense by delaying the functioning 
of TLRs in the innate immune system. ${ }^{29}$ TLR-mediated antiviral activity, along with the expression of IFN- $\beta$, interferon regulatory transcription factor 3 (IRF-3), NF-kB and ERK1/2 MAPKs, was almost completely inhibited by HBeAg, HBsAg and viral particles in HBV-treated liver non-parenchymal cells, including KCs. The mean fluorescence intensity in the immunofluorescent staining of TLR3 in monocytes from the peripheral blood of HBV infected patients was lower than in the control group. ${ }^{30}$ Along with the obvious reduction of TLR in HBeAg+ patients, pro-inflammatory cytokines and other cytokines secreted by KCs also decrease. Thus the inhibitory effect of KCs on the replication of the virus is severely reduced.

In $\mathrm{HCV}$ infection, the increase in TLRs on the surface of $\mathrm{KCs}$ and secretion of inflammatory factors increase the risk of liver damage. The activation of TLR 1-9 on non-parenchymal liver cells, including KCs, leads to the production of IFN- $\beta$ in mice, which inhibits the replication of HCV. ${ }^{31}$ Studies have shown that the level of TLR3 and TLR7 in HCV-infected patients' peripheral blood are both lower than in healthy controls, and that these levels correlate with the level of IFN- $\alpha .^{3}$ The reduction of TLRs on KCs may be closely related to immune tolerance toward and chronicity of HCV. In vitro experiments have shown that TLR7 mRNA decreases in hepatocytes infected with $\mathrm{HCV}$; after the $\mathrm{HCV}$ infection is cleared, TLR7 expression recovers. ${ }^{32}$ If a single TLR signaling pathway in KCs is continuously activated, tolerance of that pathway might be induced and its reactivity might be reduced. Chung et al. found that when they stimulated the TLR2 signaling pathway activated by HCV with ligands of TLR2 and TLR4, this led to a decrease in the IL- 6 produced by APC. ${ }^{33}$ This phenomenon shows that activation of one TLR signaling pathway can inhibit other TLR signaling pathways through negative feedback regulation, which is considered one of the mechanisms that might induce chronicity of $\mathrm{HCV}$ infection.

\section{IL-10 and TGF- $\beta$}

HBV/HCV can not only interfere with TLR signaling pathways in KCs, but also affect the secretion of $\mathrm{KC}$-derived anti-inflammatory factors, including both IL-10 and TGF- $\beta$, inducing immune tolerance in the liver. ${ }^{3}$ IL-10 is a kind of soluble protein secreted by immune cells, including KCs, and it has strong immunosuppressive properties. The development of inflammation is controlled by the concomitant production of IL-10 and TGF- $\beta$, or by nonor-low response of $\mathrm{KCs}$ to subsequent stimulation. This low-response state to PAMPs in KCs is called endotoxin tolerance, and is caused by negative regulation of the TLR signaling pathway, soluble immune regulatory molecules or even epigenetic modification. ${ }^{34}$ A higher level of IL-10 mRNA expression was detected in monocytes from chronic HCV patients. ${ }^{35}$ Studies have shown that HCV core protein induces KCs to secrete IL-10, in order to sup- press secretion of pro-inflammatory factors. ${ }^{36}$ IL-10 can also down-regulate major histocompatibility complex (MHC) class II molecules and costimulatory molecules, so interaction between KCs and NK cells, as well as their antigen-presenting function, can be suppressed. ${ }^{35}$ IL-10 can not only terminate the immune response of $\mathrm{T}$ cells, but can also suppress T cells' immune activities toward the virus. ${ }^{37}$ The direct influence of IL-10 on antigen-presenting cells can reduce the expression of the stimulating factor, inhibiting the secretion of cytokines and eventually making it hard to activate T cells. ${ }^{35}$ In an HBV-infected mouse model, when KCs were removed, the production of IL-10 and IFN- $\gamma$ decreased sharply. Researchers co-cultured KCs and $\mathrm{CD} 4+\mathrm{T}$ cells, and found that $\mathrm{KC}$ s from mice infected with HBV could induce CD4+T cells to produce IL-10 and generate Tr1-like cells. ${ }^{38}$ Thus it can be concluded that immune tolerance of $\mathrm{HBV} / \mathrm{HCV}$ is partly achieved through the secretion of IL-10 by KCs.

TGF- $\beta$ is a crucial cytokine for maintaining immune tolerance by mediating the proliferation, differentiation and survival of lymphocytes in the immune system. ${ }^{39}$ It has been reported that when stimulated by HBV, Kupffer cells produce TGF- $\beta$ instead of pro-inflammatory cytokines IL- 1 and IL- $6 .{ }^{40}$ TGF- $\beta$ can activate hepatic stellate cells (HSCs) to secrete extracellular matrix protein, which initiates cirrhosis. This phenomenon may help to answer the question of why minimal necro-inflammation is detected in chronic HBV-infected patients' liver tissue, while liver fibrosis is obvious. In addition, some studies have shown that KCs from HBV-infected mice produced TGF- $\beta$ rather than pro-inflammatory factors. ${ }^{14}$ TGF- $\beta$ can inhibit T cell proliferation by decreasing IL-2 through the SMAD3 gene and via down-regulation of cyclin D2 and cyclin E, cyclin-dependent kinase 4 (CDK4) and cmyc. ${ }^{41}$ The inhibitory effects of TGF- $\beta$ are also detected in the differentiation of $\mathrm{T}$ cells. It is safe to assume that KCs can suppress the adaptive immune response toward HBV through the production of TGF- $\beta$.

\section{The PD-1/PD-L1 signaling pathway}

Programmed cell death protein (PD-1), a crucial immune suppressive molecule, is a member of the CD28 family. PD-1 widely exists in T cells, B cells and myeloid cells, especially in activated T cells. PD-1 binds to two specific types of ligands: programmed cell death ligand 1 (PD-L1) and PD-L2. PD-L1 can be detected in non-parenchymal liver cells like sinusoidal endothelial cells and KCs. The level of PD-1, PD-L1 and PD-L2 in patients suffering chronic hepatitis is much higher than that in healthy people, and correlates with the severity of the disease. ${ }^{42}$ In infections or inflammations, KCs inhibit the replication of activated $T$ cells and their immune function through the PD-1/PD-L1 signaling pathway and interaction between cells mediated by the $\mathrm{T}$ cell immunoglobulin and mucindomain containing-3 (TIM-3) protein. An increase in ga- 
lectin-9, the ligand of TIM-3, can be detected in chronic $\mathrm{HCV}$ patients. ${ }^{43}$ Using immunofluorescence, researchers found that KCs might be a source of galectin-9, which could induce the apoptosis of $\mathrm{HCV}$ - specific cytotoxic $\mathrm{T}$ lymphocytes. A double block of TIM-3 and PD-L1/2 had a synergistic effect on the recovery of $\mathrm{HBV}$-specific $\mathrm{CD} 8+$ T cells. ${ }^{43}$ A high level of PD-1 expression was found in apoptotic $\mathrm{T}$ lymphocytes; the number, antiviral effect and immune function of $\mathrm{T}$ lymphocytes were obviously promoted when the PD-1/PD-L1 signaling pathway was blocked by a PD-L1 antibody. Thus it may be concluded that negative regulation of the replication and immune function of $\mathrm{T}$ lymphocytes depends on the activation of the PD-1/PD-L1 signaling pathway by the binding of PDL1 expressed by KCs and PD-1 expressed by T lymphocytes. HBV's escape from host immune surveillance, the chronicity of $\mathrm{HBV}$ infection and immune tolerance are closely related to the PD-1/PD-L1 signaling pathway. Although it has been stated that $\mathrm{HCV}$ core protein can induce PD-L1 expression, it is not clear whether it is caused by the direct influence of the virus or the negative feedback system induced by continuous infection. ${ }^{3}$

\section{The Fas/FasL system}

The Fas/FasL system is the mechanism that mainly mediates cell apoptosis. The apoptosis of a host's cells is a crucial part of the pathogenesis of viral infection. When cells are infected with HBV, the abnormality in the expression and distribution of Fas/FasL leads to host cell apoptosis; thus, hepatocytes are damaged. When the body is infected with HBV, a high expression of Fas is detected in hepatocytes, and KCs promote the secretion of FasL. The higher the expression rate of Fas/FasL is, the higher the degree of inflammation and tissue damage. ${ }^{44}$

The Fas/FasL system is a double-edged sword in the immune system of the liver. On the one hand, FasL expressed by KCs can bind to Fas expressed by hepatocytes infected with HBV to promote apoptosis of the infected hepatocytes, contributing to the clearance of $\mathrm{HBV}^{45}$ On the other hand, Fas is also expressed on lymphocytes, thus apoptosis of hepatocytes is accompanied by the apoptosis of lymphocytes, which can devastate specific humoral and cellular immunity. ${ }^{46}$ Unfortunately the latter effect can be stronger than the former. Fas/FasL-mediated apoptosis of lymphocytes is beneficial to immune tolerance, so KCs may induce tolerance of $\mathrm{HBV}$ partly though the Fas/FasL system.

\section{The NLRP3 inflammasome}

HBV/HCV can activate the NLRP3 inflammasome, and at the same time they also inhibit the activity of the NLRP3 inflammasome in KCs, suppressing their secretion activity. Negash et al. found that KCs can secrete IL-1 $\beta$, which induces a variety of pro-inflammatory mediators via cas- pase-1 or the NLRP3 inflammasome in KCs, eventually causing inflammation and fibrogenesis. The maturation of IL-1 $\beta$ induced by HCV was suspended in caspase- 1 or NLRP3 knockdown mice. ${ }^{47}$ The HCV virus can also block IL-1 $\beta$ directly by suppressing the activity of NF- $\mathrm{kB}$, so the secretion of pro-inflammatory factors by KCs is severely inhibited. In patients infected with HCV, the level of IL-1 $\beta$ and caspase- 1 in KCs decreases significantly after they are treated with pyrrolidineditiocarbamate and/or bafilomycin A. ${ }^{48}$ The increase in the NLRP3 inflammasome mRNA in the livers of chronic HBV patients illustrates the up-regulation of NLRP3 expression in KCs, which has been proven by immunohistochemistry. ${ }^{49}$ As a result, $\mathrm{HBV} / \mathrm{HCV}$ tolerance might be induced by blocking IL-1 $\beta$ through suppression of the NLRP3 inflammasome in KCs.

\section{Conclusions}

Due to the dual role of $\mathrm{KCs}$ in the immune response to $\mathrm{HBV} / \mathrm{HCV}$, it is exceedingly difficult for researchers to elucidate the detailed mechanisms involved. Studies on the role of $\mathrm{KCs}$ in viral hepatitis are beneficial to the body of knowledge on this disease, and can help us not only to get a better understanding of the pathogenesis of $\mathrm{HBV} / \mathrm{HCV}$, but also to find potential therapeutic targets that are theoretically possible. For example, researchers found they could prevent the exhausting of $\mathrm{T}$ lympho-

Fig. 1. Negative feedback regulation of the TLR signaling pathways exists in KCs. Activation of one specific TLR signaling pathway can inhibit other TLR signaling pathways. Anti-inflammatory cytokines IL-10 and TGF- $\beta$ secreted by KCs can inhibit immune cells, including NK and T cells, in the immune response to HBV/HCV. PD-L1 and FasL produced by KCs can induce the apoptosis of T cells and B cells, which eventually leads to immune tolerance to HBV/HCV. FasL can also lead to the apoptosis of $\mathrm{HBV} / \mathrm{HCV}$-infected hepatocytes, facilitating the clearance of HBV/HCV

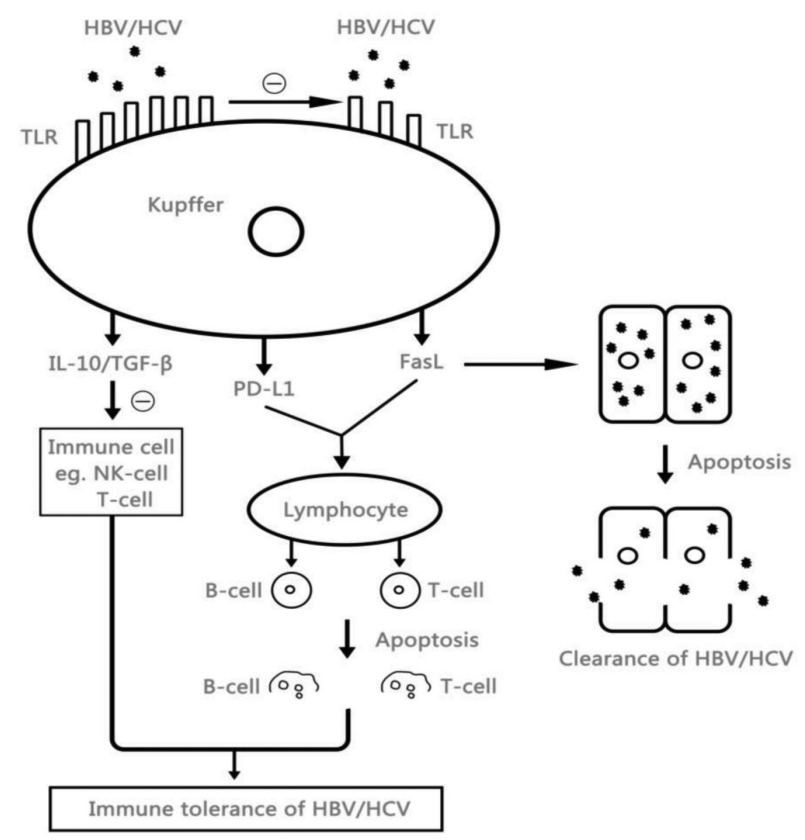


cytes and promote the clearance of the HBV/HCV virus by blocking IL-10 receptors. Mechanisms that can be carried out just by blocking a single factor will undoubtedly bring promising progress to the treatment of $\mathrm{HBV} / \mathrm{HCV}$. The effect of the PD/PD-L1 signaling pathway is to suppress the immune function of HBV specific T cells, which is crucial to the chronicity of HBV infection. Interfering with this signaling pathway can provide significant therapeutic benefits. It is an inescapable conclusion that $\mathrm{KCs}$ are the hinge of the immune response toward $\mathrm{HBV} / \mathrm{HCV}$ infection, so more effort should be devoted to elucidating the role of $\mathrm{KCs}$ in the pathogenesis of $\mathrm{HBV} / \mathrm{HCV}$.

\section{References}

1. Vassilopoulos D, Calabrese LH. Management of rheumatic disease with comorbid HBV or HCV infection. Nat Rev Rheumatol. 2012;8(6):348-357.

2. Strauss O, Dunbar PR, Bartlett A, Phillips A. The immunophenotype of antigen presenting cells of the mononuclear phagocyte system in normal human liver - a systematic review. $J$ Hepatol. 2015;62(2):458-468.

3. Boltjes A, Movita D, Boonstra A, Woltman AM. The role of Kupffer cells in hepatitis B and hepatitis C virus infections. J Hepatol. 2014;61(3):660-671.

4. Khakoo SI, Soni PN, Savage K, et al. Lymphocyte and macrophage phenotypes in chronic hepatitis $C$ infection. Correlation with disease activity. Am J Pathol. 1997;150(3):963-970.

5. Boltjes A, van Montfoort N, Biesta PJ, et al. Kupffer cells interact with hepatitis B surface antigen in vivo and in vitro, leading to proinflammatory cytokine production and natural killer cell function. J Infect Dis. 2015;211:1268-1278.

6. Movita D, Kreefft K, Biesta $P$, et al. Kupffer cells express a unique combination of phenotypic and functional characteristics compared with splenic and peritoneal macrophages. J Leukoc Biol. 2012;92(4):723-733.

7. Taylor PR, Martinez-Pomares L, Stacey M, Lin HH, Brown GD, Gordon S. Macrophage receptors and immune recognition. Annu Rev Immunol. 2005;23:901-944.

8. Dominguez-Soto A, Aragoneses-Fenoll L, Gomez-Aguado F, et al. The pathogen receptor liver and lymph node sinusoidal endotelial cell C-type lectin is expressed in human Kupffer cells and regulated by PU.1. Hepatology. 2009;49(1):287-296.

9. Huang YW, Lin SC, Wei SC, et al. Reduced Toll-like receptor 3 expression in chronic hepatitis $B$ patients and its restoration by interferon therapy. Antivir Ther. 2013;18(7):877-884.

10. Imaizumi T, Sashinami H, Mori F, et al. Listeria monocytogenes induces the expression of retinoic acid-inducible gene-I. Microbiol Immunol. 2006;50(10):811-815.

11. Vanlandschoot P, Van Houtte F, Roobrouck A, et al. LPS-binding protein and CD14-dependent attachment of hepatitis B surface antigen to monocytes is determined by the phospholipid moiety of the particles. J Gen Virol. 2002;83(Pt 9):2279-2289.

12. Op den Brouw ML, Binda RS, Geijtenbeek TB, Janssen HL, Woltman AM. The mannose receptor acts as hepatitis B virus surface antigen receptor mediating interaction with intrahepatic dendritic cells. Virology. 2009;393(1):84-90.

13. Chang S, Dolganiuc A, Szabo G. Toll-like receptors 1 and 6 are involved in TLR2-mediated macrophage activation by hepatitis C virus core and NS3 proteins. J Leukoc Biol. 2007;82(3):479-487.

14. Hosel M, Quasdorff $M$, Wiegmann $K$, et al. Not interferon, but interleukin-6 controls early gene expression in hepatitis B virus infection. Hepatology. 2009;50(6):1773-1782.

15. Phillips S, Chokshi S, Riva A, Evans A, Williams R, Naoumov NV. CD8(+) T cell control of hepatitis $B$ virus replication: direct comparison between cytolytic and noncytolytic functions. J Immunol. 2010;184(1):287-295.

16. Tu Z, Pierce RH, Kurtis J, Kuroki Y, Crispe IN, Orloff MS. Hepatitis C virus core protein subverts the antiviral activities of human Kupffer cells. Gastroenterology. 2010;138(1):305-314.
17. Hosomura $\mathrm{N}$, Kono H, Tsuchiya M, et al. HCV-related proteins activate Kupffer cells isolated from human liver tissues. Dig Dis Sci. 2011;56(4):1057-1064.

18. Fletcher NF, Sutaria R, Jo J, et al. Activated macrophages promote hepatitis $C$ virus entry in a tumor necrosis factor-dependent manner. Hepatology. 2014;59(4):1320-1330.

19. Movita D, van de Garde MD, Biesta P, et al. Inflammatory monocytes recruited to the liver within 24 hours after virus-induced inflammation resemble Kupffer cells but are functionally distinct. J Virol. 2015;89(9):4809-4817.

20. Seeger $P$, Bosisio D, Parolini $S$, et al. Activin $A$ as a mediator of NK-dendritic cell functional interactions. J Immunol. 2014;192(3): 1241-1248.

21. Mundt B, Kuhnel F, Zender L, et al. Involvement of TRAIL and its receptors in viral hepatitis. FASEB J. 2003;17(1):94-96.

22. Woltman AM, Boonstra A, Janssen HL. Dendritic cells in chronic viral hepatitis B and C: Victims or guardian angels? Gut. 2010;59(1):115-125.

23. Heymann F, Peusquens J, Ludwig-Portugall I, et al. Liver inflammation abrogates immunological tolerance induced by Kupffer cells. Hepatology. 2015;62(1):279-291.

24. Sandler NG, Koh C, Roque A, et al. Host response to translocated microbial products predicts outcomes of patients with HBV or HCV infection. Gastroenterology. 2011;141(4):1220-1230.

25. Nakamoto N, Kanai T. Role of toll-like receptors in immune activation and tolerance in the liver. Front Immunol. 2014;5:221.

26. Visvanathan K, Skinner NA, Thompson AJ, et al. Regulation of tolllike receptor-2 expression in chronic hepatitis $B$ by the precore protein. Hepatology. 2007;45(1):102-110.

27. Wang S, Chen Z, Hu C, et al. Hepatitis B virus surface antigen selectively inhibits TLR2 ligand-induced IL-12 production in monocytes/macrophages by interfering with JNK activation. J Immunol. 2013;190(10):5142-5151.

28. Wu J, Lu M, Meng Z, et al. Toll-like receptor-mediated control of $\mathrm{HBV}$ replication by nonparenchymal liver cells in mice. Hepatology. 2007;46(6):1769-1778.

29. Fullard N, O'Reilly S. Role of innate immune system in systemic sclerosis. Semin Immunopathol. 2015;37(5):511-517.

30. Thomson AW, Knolle PA. Antigen-presenting cell function in the tolerogenic liver environment. Nat Rev Immunol. 2010;10(11):753-766.

31. Broering $\mathrm{R}, \mathrm{Wu} \mathrm{J}$, Meng $\mathrm{Z}$, et al. Toll-like receptor-stimulated nonparenchymal liver cells can regulate hepatitis $C$ virus replication. J Hepatol. 2008;48(6):914-922.

32. Chang S, Kodys K, Szabo G. Impaired expression and function of toll-like receptor 7 in hepatitis $C$ virus infection in human hepatoma cells. Hepatology. 2010;51(1):35-42.

33. Chung $H$, Watanabe $T$, Kudo $M$, Chiba T. Hepatitis $C$ virus core protein induces homotolerance and cross-tolerance to toll-like receptor ligands by activation of toll-like receptor 2 . J Infect Dis. 2010;202(6):853-861.

34. Mengshol JA, Golden-Mason L, Arikawa T, et al. A crucial role for Kupffer cell-derived galectin-9 in regulation of T cell immunity in hepatitis C infection. PLoS One. 2010;5(3):e9504.

35. Wilson EB, Brooks DG. The role of IL-10 in regulating immunity to persistent viral infections. Curr Top Microbiol Immunol. 2011;350:39-65.

36. Lian ZR, Xu YF, Wang XB, Gong JP, Liu ZJ. Suppression of histone deacetylase 11 promotes expression of IL-10 in Kupffer cells and induces tolerance following orthotopic liver transplantation in rats. J Surg Res. 2012;174(2):359-368.

37. Xu L, Yin W, Sun R, Wei H, Tian Z. Kupffer cell-derived IL-10 plays a key role in maintaining humoral immune tolerance in hepatitis $B$ virus-persistent mice. Hepatology. 2014;59(2):443-452.

38. Xu L, Yin W, Sun R, Wei H, Tian Z. Liver type I regulatory T cells suppress germinal center formation in HBV-tolerant mice. Proc Natl Acad Sci USA. 2013;110(42):16993-16998.

39. Li MO, Wan YY, Sanjabi S, Robertson AK, Flavell RA. Transforming growth factor-beta regulation of immune responses. Annu Rev Immunol. 2006;24:99-146.

40. Li H, Zheng HW, Chen $H$, et al. Hepatitis B virus particles preferably induce Kupffer cells to produce TGF-beta1 over pro-inflammatory cytokines. Dig Liver Dis. 2012;44(4):328-333.

41. Cerwenka A, Swain SL. TGF-beta1: Immunosuppressant and viability factor for T lymphocytes. Microbes Infect. 1999;1(15):1291-1296. 
42. Chen J, Wang XM, Wu XJ, et al. Intrahepatic levels of PD-1/PD-L correlate with liver inflammation in chronic hepatitis B. Inflamm Res. 2011;60(1):47-53.

43. Larsson M, Shankar EM, Che KF, et al. Molecular signatures of T-cell inhibition in HIV-1 infection. Retrovirology. 2013;10:31.

44. Lee JY, Chae DW, Kim SM, et al. Expression of FasL and perforin/ granzyme B mRNA in chronic hepatitis B virus infection. J Viral Hepat. 2004;11(2):130-135.

45. Liu W, Lin YT, YanXL, et al. Hepatitis B virus core protein inhibits Fasmediated apoptosis of hepatoma cells via regulation of $\mathrm{mFas} / \mathrm{Fas} \mathrm{L}$ and sFas expression. FASEB J. 2015;29(3):1113-1123.

46. Chen, Y, Liu Z, Liang S, et al. Role of Kupffer cells in the induction of tolerance of orthotopic liver transplantation in rats. Liver Transpl. 2008;14(6):823-836

47. Negash AA, Ramos HJ, Crochetn N, et al. IL-1beta production through the NLRP3 inflammasome by hepatic macrophages links hepatitis $C$ virus infection with liver inflammation and disease. PLoS Pathog. 2013;9(4):e1003330.

48. Burdette D, Haskett A, Presser L, McRae S, Iqbal J, Waris G. Hepatitis $C$ virus activates interleukin-1beta via caspase-1-inflammasome complex. J Gen Virol. 2012;93(Pt 2):235-246.

49. Csak T, Ganz M, Pespisa J, Kodys K, Dolganiuc A, Szabo G. Fatty acid and endotoxin activate inflammasomes in mouse hepatocytes that release danger signals to stimulate immune cells. Hepatology. 2011;54(1):133-144. 